\title{
Update
}

\section{QUANTUM COSMOLOGIES AND THE "BEGINNING"}

\author{
by Willem B. Drees
}

\begin{abstract}
The cosmology proposed by Stephen Hawking has been understood as support for an atheistic stance, due mainly to its view of the nature of time in combination with the absence of explicit boundary conditions. Against such a view, this article argues that one might develop a theistic understanding of the Universe in the context of Hawking's cosmology. In addition, the quantum cosmologies of Andrej Linde and Roger Penrose are presented. The coexistence of different research programs and their implicit metaphysical views about the nature of quantum reality and time may have profound implications for philosophy and theology.
\end{abstract}

Keywords: Big Bang theory; creatio ex nihilo; S. W. Hawking; A. D. Linde; R. Penrose; quantum cosmology.

.. . a universe with no edge in space, no beginning or end in time, and nothing for a Creator to do.

$$
\text { -Carl Sagan }(1988, x)
$$

Carl Sagan interprets the recent cosmology developed by Stephen Hawking as support for an atheistic stance. I will argue, however,

Willem B. Drees is a staff member of the Interdisciplinary Centre for the Study of Science, Society, and Religion (Bezinningscentrum) at the Free University, De Boelelaan $1115,1081 \mathrm{HV}$ Amsterdam, the Netherlands. His investigations were supported by the Foundation for Research in the Field of Theology and the Science of Religions in the Netherlands (STEGON), which is subsidized by the Netherlands Science Organization $(N W O)$. The resulting thesis was published in 1990 by Open Court (LaSalle, Illinois), and the present article is a modified version of one of its chapters. The author received a Fulbright grant and additional support from various Dutch foundations that made it possible to study at the Center for Theology and the Natural Sciences in Berkeley, California, and the Chicago Center for Religion and Science. The author expresses his gratitude to his thesis advisers, $R$. Hensen and $H$. van Woerden at the University of Groningen (the Netherlands), and to R. J. Russell (CTNS, Berkeley) and P. Hefner (CCRS, Chicago).

[Zygon, vol. 26, no. 3 (September 1991).]

(C) 1991 by the Joint Publication Board of Zygon. ISSN 0591-2385 
against Sagan, that Hawking's cosmology does allow for a theistic understanding of the world as God's creation. I will also point to other research programs in cosmology and argue that, through their content and coexistence, they may have implications for different views of theology and science.

After this article reviews some limitations of the Big Bang theory, a few recent cosmologies will be presented, with emphasis on the "beginning," time, and quantum reality. 'The article concludes with some implications for philosophy and theology and with an interpretation of creatio ex nihilo in the context of Hawking's cosmology.

\section{LIMITS OF THE BIG BANG THEORY}

The Big Bang is the accepted theory about the evolution of the Universe; however, it is often misunderstood as dealing with an initial explosion. As expressed in the title of Steven Weinberg's popular exposition, The First Three Minutes (1977), it seems as if the Universe has a beginning and as if we can describe the processes right from the start. Nevertheless, a closer look reveals certain problems with the second claim: our capability of describing the Universe from the very start and even uncertainty about the first claim, the beginning itself.

The Big Bang theory is a combination of two different theories, general relativity about space-time and quantum theories about matter. This combination implies three limits to the Big Bang theory:

1. Current theories about matter are only valid up to a finite temperature and, hence, only after the first fraction of a second after the Singularity, the initial moment that follows from general relativity. Further speculations have to deal with temperatures and densities for which the relevant particle physics is not yet well established.

2. Closer to the Singularity comes a moment, presumably the Planck time (a number constructed from the fundamental constants of quantum theory and gravity, about $10^{-43}$ seconds after the initial Singularity in the Big Bang model), when general relativity must be replaced by a quantum theory of gravity. Before the Planck time the theories about space and time are not known; even the meaningfulness of space and time is uncertain (which signals trouble: once time is no longer meaningful, it becomes unclear what can be meant by before).

3. The initial Singularity itself is a third limit, if there is an initial Singularity. Thus the Big Bang theory results in unrealistic numbers, 
such as an infinite density. It has been shown that such singularities are unavoidable in general relativity, once certain very general assumptions are made, like causality and a positive energy density (Hawking and Penrose 1970). However, general relativity itself is probably invalid for moments before the Planck time. Whether there is a singular moment in a theory of quantum gravity which is to supersede general relativity cannot be decided a priori, before such a theory of quantum gravity has been proposed.

The first and second limits are clearly limits to our present knowledge; the third limit, the Singularity, seems an edge of reality, but it is "hidden" behind the other two.

To describe our Universe we need, in addition to physical laws, some initial conditions, numbers which are not explained by the theory but are assumed-for example, the density. The density turns out to be very close to the critical density, which is the boundary between an infinitely expanding (open) and a recollapsing (closed) universe. This leads to a question, called the flatness problem: Why is the density of our Universe so close to that density which corresponds to a "flat universe"? Such initial conditions are even more peculiar than they seem as a consequence of the horizon problem: regions that we observe in different directions have not been in causal contact during the whole history of the Universe, if one accepts the Big Bang theory, and yet they have the same density.

Besides those initial conditions, the Big Bang theory also assumes certain general features of the Universe: the laws of physics, the three spatial dimensions and the one dimension of time, and even its very existence.

Developments beyond the Big Bang theory do not mean that there is anything wrong with the theory. "In sum, all the available evidence indicates that the standard cosmology provides an accurate accounting of the evolution of the Universe from $0.01 \mathrm{sec}$ after the bang [Singularity] until today, some 15 or so Byr [Billion years] later-quite a remarkable achievement!' (Turner 1985, 271). None of the scientists discussed below objects to the theory, within its limits. The problems and assumed initial conditions aren't inconsistencies but exhibit the limitations of the explanatory power of the Big Bang theory. The challenge for physicists has been to explain the assumptions (laws, initial conditions) and to extend the domain of validity of the theories. Three different approaches will be discussed, each by referring to the work of one major cosmologist: Andrej Linde, Stephen Hawking, and Roger Penrose. 


\section{ANDREJ Linde: ETernal GhaOtic Cosmology}

The performance is still going on, and it will continue eternally. In different parts of the universe different observers see its endless variations.

-Andrej Linde (1987b, 68)

Andrej Linde, a Russian cosmologist, insists that the Universe underwent a period of rapid expansion-a so-called inflation. ${ }^{2}$ His version is called chaotic inflation. Chaotic cosmologies try to explain such features as homogeneity, which we find special about our Universe, as the consequence of natural processes, without requiring any special initial conditions. Earlier versions of chaotic cosmology emphasized the role of dissipative processes, which would smear out inhomogeneities and produce, after billions of years, a universe which looks as homogeneous as the Universe we observe. Imagine a beach where children have built castles from sand and another beach which remains untouched; after a few days of winds and tides, the two beaches look similar. Almost all initial conditions produce similar results if dissipative processes are sufficiently effective.

Linde does not emphasize dissipative processes; rather, he proposes that the conditions for inflation have been satisfied in some regions (domains) of the Universe while not, or later, in other domains. Domains that inflate form very large bubbles. Sometimes these are called miniuniverses, but mini should not be misunderstood; these domains are supposed to be much larger than the observable Universe. Each miniuniverse would resemble a Big Bang universe, quite homogeneous and of the right density. However, the Universe at large would not be homogeneous, but a cluster of bubbles, or miniuniverses, attached to each other.

Those bubble universes need not have the same properties. By analogy, the physics for water and ice is of course very different although the fundamental, underlying theory is the same. In cosmology, the underlying theory need not be based upon three spatial dimensions and one dimension of time. It might be ten- or eleven-dimensional. Some of those dimensions might "compactify" - become very small. We observe the four "large" dimensions, whereas the others are unobservable as spatial dimensions, although their presence might be effective as interactions (forces) in a world described in terms of the large dimensions. There might be more than one way in which the higher-dimensional underlying structure might compactify and, hence, produce different bubbles with different numbers of dimensions and physical laws. 
No Beginning. Bubble formation goes on; the universe "unceasingly reproduces itself and becomes immortal" (Linde 1987b, 66). People and miniuniverses die, but the show goes on. It "no longer seems necessary to assume that there actually was some first mini universe appearing from nothing or from an initial singularity at some moment $t=0$ before which there was no space-time at all", (Linde 1987b, 66). This solves "the singularity problem. The problem is not the existence of singularities in the Universe, but the statement (or common belief) that the Universe does not exist eternally and that there exists 'some time at which there is no spacetime at all' " (Linde 1987a, 606). ${ }^{3}$

Linde's approach is mostly formulated in evolutionary terms-for instance, "inflation" as spatial expansion in time. However, he also holds that there is no evolution in time for the Universe as a whole. "What we measure is phenomenological time, which can be introduced by a local observer inside a long-living virtual miniuniverse. . . An eastern philosopher would say that the absolute does not evolve in time, but the virtual apparent world is observed as being time-dependent' (Linde 1985, 289; see also Linde 1989, 355). This time is not strictly restricted to the inside of a bubble. "Any two points of such a universe in a sufficiently distant past could be causally connected and corresponding observers could synchronise their clocks even though later they may live in mini universes which have become causally disconnected due to the exponential expansion of the universe"' (Linde 1987a, 620).

Existence of All Possibilities. The conditions for different mini universes appear by chance. Some domains inflate to a more significant long-lived status, which gives, as Linde acknowledged (1987b, $66)$, a Darwinian flavor to his cosmology. There are mutations, as subsequent bubbles may be different-for instance, in dimensionality. Selection finds its parallel in inflation, which gives a long life to a bubble. Linde combines the idea of selection operating on chance mutations with another, rather different but in my opinion more fundamental, philosophical idea: the realization of all possibilities. For instance, all possible dimensionalities and phenomenological laws of physics "should exist in different domains of the universe" (Linde 1987a, 623). "One may say therefore that not only could God create the universe differently, but in $\mathrm{His}$ wisdom $\mathrm{He}$ created a universe which has been unceasingly producing different [mini-] universes of all possible types" (Linde 1987a, 607).

Even though all possible bubbles in Linde's cosmology become actual in the eternity of time available, this is only what is possible 
within his cosmology. Whether God has any options besides such an eternal chaotic universe, with all its bubbles, is the form the uniqueness question takes at this level. Uniqueness is not settled by saying that all possibilities of this theory are realized; it would be necessary to say whether any other theories are possible. Let us therefore look at other proposals, such as the quantum cosmology of Hawking.

\section{STEPHEN HAWKing: TIMEless QuANTUM COSMOLOGY}

I think that the initial conditions are as suitable a subject for scientific investigation as are the local physical laws. We shall not have a complete theory until we can do more than merely say that "things are as they are because they were as they were."

-Stephen Hawking (1980), in Boslaugh (1985, 133)

Stephen Hawking, a famous English cosmologist, titled his inaugural lecture for the Lucassian chair (Newton's chair) at Cambridge Is the End in Sight for Theoretical Physics? According to him, physics is about to discover and explain the most fundamental laws, as well as the ultimate boundary conditions for the Universe. This is against the standard view, which sees initial conditions, or boundary conditions, as given-as the way a situation has been set up. Ordinary physics explains a collision of billiard balls, given their initial positions and velocities. Hawking holds that physics can, when considering the whole, do without such an unexplainable given. He proposed in 1983, in collaboration with James Hartle, a quantum cosmology that seems to do without initial (or boundary) conditions.

In the Big Bang theory the state of a universe at one time can be calculated on the basis of the Einstein equations and at another time as a boundary condition. In that sense, one could say that one state "arises out of" another state. One could visualize this as a pancakea slice of time with the two large surfaces representing the initial and the final state in the calculation. (Each surface in this image stands for the three-dimensional space with material content at a given moment; the pancake is supposed to be a four-dimensional "volume.")

The Hartle-Hawking proposal does without such another state. The probability of one state is calculated while that state is the only boundary. (We have to deal with probabilities since the proposal is in the context of quantum physics.) Instead of a pancake with two surfaces, as suggested for the standard view, one could think of a single closed surface, such as the surface of a sphere, representing 
only one state. There would be no need for another boundary in the calculation; a compact surface, like that of a sphere, has no boundaries even though it is finite. As Hawking has formulated it, the boundary condition for the Universe is that it has no boundary.

However, requiring compactness or even the absence of a boundary is in a sense a boundary condition-although not of properties of fields on the boundary but rather of the properties of the boundary itself. Hawking's proposal is certainly of an attractive simplicity, but other metaphysical views might lead to other proposals for the boundary condition. For example, Frank Tipler proposed the "Teilhard boundary condition," which is essentially an idea about the future boundary (Tipler 1989, 239). And Alexander Vilenkin $(1986,3568)$ proposed a boundary condition based on causality, an idea that seems similar in spirit to the cosmology of Roger Penrose, who assumes a fundamental difference between initial and final singularities (see below).

Hawking's "trick" has its repercussions. Ordinary time, with the notion of becoming, disappears. One could still see a trace of the original time variable in the internal four-dimensional space which fits at the three-dimensional boundary, but it is an imaginary time variable, and thereby the time dimension is completely on a par with the spatial dimensions. There is no reason to call the fourth dimension a time variable at this level. ${ }^{4}$ The calculations are complicated, and certainly so if one includes all fields that should be part of a realistic theory. No model describing our Universe exists yet, but there are some indications that the density-at least in a simplified model-should be close to the critical density (Hawking and Page 1986 ) and that most universes of this type have an inflationary phase (Gibbons, Hawking, and Stewart 1987).

One can still reconstruct the notion of time by changing to another description, since the Hartle-Hawking cosmology has two levels of calculation. The approach described, which is the unique feature of this proposal, does not refer to any other state in calculating the probability of one state. In that sense the theory is a timeless description of the states of the Universe. But one can consider two states at different moments, so thus this is a description from within time. To reconstruct this description from the timeless perspective, the notion of time has to be introduced, and the three-dimensional spaces have to be ordered in a time sequence. Time can be defined on the basis of the fields and the geometry of the states. In that sense, time is a phenomenological construct. (Something similar is possible in a Big Bang universe, where time can be defined on the basis of the temperature of the background radiation or on the curvature.) 
In the timeless approach, one can calculate the probabilities of all of the three-dimensional spaces and then line them up in stacks which resemble four-dimensional space-times, like our Big Bang Universe. It turns out that those sequences behave according to an equation which describes their evolution-if the system is described from within time.

Compared with the Big Bang theory, there are two important qualifications:

1. This theory does not produce one stack, that is, one Big Bang Universe. The three-dimensional spaces form a variety of such stacks, each stack corresponding to one particular evolutionary sequence of three-dimensional geometry and matter configurations in a universe.

2. Not all spaces fit in a stack, and hence not all can be interpreted as being part of an evolutionary sequence. The theory works well for the states that reproduce the classical theory. "But the more "quantum mechanical' . . . the state, the harder it becomes to sustain an interpretation of anything 'evolving' in time. In effect, the concept of 'spacetime' only has an unambiguous meaning within the framework of non-quantum physics, whereas the idea of threedimensional 'space' can be applied to both the quantum and the classical theories'" (Isham 1988, 397).

In summary, in this theory the timeless level of description is more fundamental than the description from within time.

"Beginning" as a Feature of the Description. This theory avoids the problem of an initial Singularity, a $t=0$, as in the Big Bang theory. Time near the Singularity is unlike our ordinary time. If one uses the Hartle-Hawking scheme for spaces which are close to the "beginning' of the Big Bang Universe (i.e., small spaces), one would expect to approach the interesting $t=0$ moment. However, "the phenomenological time begins to pick up something like an imaginary part with its associated non-physical features. By this means, the problem of the 'beginning of time' is adroitly averted"' (Isham 1988, 400). In this sense, there is no initial Singularity where the theory breaks down. Only our interpretation, in terms of our usual notion of time, breaks down.

A more visual way to present it has been used by Hawking. There is a beginning of the coordinate, but no edge-as the North Pole is an extreme of our coordinates based on degrees of latitude, but not an edge of the globe. "Time ceases to be well defined in the very early universe just as the direction 'north' ceases to be well defined at the North Pole of the Earth. . . . The quantity that we measure as time 
had a beginning, but that does not mean spacetime has an edge, just as the surface of the Earth does not have an edge at the North Pole", (Hawking 1984b, 358, and 1984c, 14). As the space-time is compact, according to his proposal, a past or future infinity is impossible. "Thus, in this sense, the universe will have a beginning and an end" (Hawking 1987, 650). However, that need not be a Singularity in the sense of a breakdown of the laws of physics.

Hawking points to the similarity between his view and the view of Augustine,${ }^{5}$ who introduced in the Christian tradition the notion of creation with time, in which time is part of the created order. According to Hawking, "time is just a coordinate that labels events in the universe. It does not have any meaning outside the spacetime manifold. To ask what happened before the universe began is like asking for a point on Earth at $91^{\circ}$ north latitude; it just is not defined. Instead of talking about the universe being created, and maybe coming to an end, one should just say: The universe is"' (Hawking 1987, 651).

There is, of course, a very important difference between Augustine and Hawking. Augustine no doubt understood the beginning of time simultaneously with the creation as an event outside the scope of natural knowledge. But Hawking holds it to be only the beginning of a coordinate, without reference to any special event and without a breakdown of physical describability. For Hawking, this has theological implications. "So long as the universe had a beginning, we could suppose it had a creator. But if the universe is really completely self-contained, having no boundary or edge, it would have neither beginning nor end: it would simply be. What place, then, for a creator?" (Hawking 1988, 141).

\section{Roger Penrose: Time-Asymmetric Realist COSMOLOGY}

My own line of argument has been driven, in part under the sting of Hawking's penetrating criticism, into some dangerous but fascinating territory.

--Roger Penrose $(1981,244)$

Roger Penrose, a mathematician and cosmologist from Oxford, proved in the late 1960s, together with Hawking, theorems about the presence of singularities in wide classes of physically reasonable solutions of the equations of general relativity (Hawking and Penrose 1970). However, he has taken another path than Hawking with respect to time, the nature of quantum reality, and the specialness of the universe. Those issues form the heart of the "dangerous but fascinating territory.",6 
Specialness of the Initial State. According to Hawking, the initial conditions of the Universe are the only ones that are possible; he does not allow variety. Linde argues that all possibilities happen from time to time. Penrose, who argues that the initial state was very special, based his argument on entropy. The observed entropy per baryon (proton, neutron) is about $10^{8}$, and there are about $10^{80}$ baryons in the observable Universe. Hence the total entropy of the observable Universe is $10^{88}$. This seems enormous but is, according to Penrose, incredibly low. If all the mass would have been clustered in solar-size black holes, the entropy per "baryon" would have been $10^{20}$, and hence the total entropy would have been $10^{100}$. If the whole observable Universe consisted of only one black hole, its entropy would be $10^{123}$. "This provides us with a measure of the degree to which the initial state was special" (Penrose 1981, 248).

Penrose considers an anthropic explanation: could the observed (or inferred) low entropy be explained as a requirement for life and consciousness? In his view, such an explanation fails. A universe with an entropy of $10^{115}$ would have fitted as well, and it would have been "a vastly 'cheaper' method than the one which appears actually to have been used" (Penrose 1981, 254). "Indeed, it would appear from this that the Creator was not particularly 'concerned' about our existence, but was constrained in some very precise time-asymmetric way for some quite other reason. From this point of view, our present existence would arise merely as a byproduct!' (Penrose 1981, 255).

Time Asymmetry. If time were reversible, the beginning of the Universe would have to be like the end. In Penrose's view, time is asymmetric, which is in accord with his emphasis on the specialness of the initial conditions. Penrose accepts that all the laws of ordinary physics are time-symmetrical; even the standard explanation that the asymmetry we observe is an effect of the low entropy of the initial conditions. But that leaves the question why those initial conditions were so special. Penrose holds that there must be a law which severely restricts initial singularities. He conjectures that this law would imply the absence of gravitational clumping in initial states. Although it looks like an initial condition, he presents it as a new law, which should hold for all initial singularities.

The asymmetry of time is, for Penrose, not manifest only in the initial conditions. He lists seven "arrows of time," most of which can be traced back to the specialness of the initial conditions. For Hawking, the time symmetry of the theory is beyond doubt, and the apparent time asymmetry of the observations needs to be explained away. For Penrose it is the opposite: the experience of time asym- 
metry is taken as beyond doubt and hence the theory should be changed to incorporate it. "The puzzle then becomes: why does Nature choose to hide this time-asymmetry so effectively?' '(Penrose 1979, 638). Another hidden asymmetry can be found in biology. Almost all animals on Earth are externally symmetric with respect to their left and right sides; however, the DNA molecules which guide the growth of these symmetric beings are all asymmetric, and all in the same way (Penrose 1979,638).

Quantum Reality. Quantum theories are successful, but their interpretation is still subject to discussion. The state vector (or, equivavalently, the wave function) might describe the system as being in a combination of a plurality of states, say an electron with spin up or down, or a particle going through gate A or gate B. This is called a linear superposition, as the different possibilities are added in the state vector without interfering with one another. Some tried to escape this view of reality by claiming that the superposition merely reflects our ignorance ("hidden variables"). This has been shown to imply that one must give up on physics as describing local interactions which do not immediately affect the state of the system at other places. One could perhaps live with the superposition as real at a submicroscopic, quantum level; but it carries over to macroscopic objects. Penrose rejects the famous thought experiment of Schrödinger's cat in a superposition of being both dead and alive. "Surely, at that level the cat is either dead or alive" (Penrose 1987a, 32 ). The tension between the many possibilities and the (apparently) one actuality lends itself to a wide variety of interpretations.

The Many Worlds Interpretation, first proposed by Everett (1957; see also DeWitt and Graham 1973), maintains the superposition throughout. All possible outcomes of measurements are actual outcomes. This might be interpreted as a branching of the world into many "worlds" with slight differences, with, say, the spin of an electron along a vertical or a horizontal axis. Since any process with different possible outcomes implies splitting, this view seems to lead to an enormous number of "universes," coexisting without interacting. However, Tipler has argued forcefully that "everything else" need not split with the property considered; quantum transitions on distant stars do not induce splits in human beings. ${ }^{7}$ In any event, the many worlds idea eliminates the tension between potentiality and actuality by equating them.

The idea of superposition at macroscopic levels has been criticized heavily (e.g., Bell 1981, Stein 1984, Healey 1984, Shimony 1986). Indeed, Penrose assumes that there is no superposition for 
macroscopic bodies, although the principle is valid at the quantum level. Rejecting the transfer of superpositions from the quantum level to the macroscopic level implies that there has to be a reduction of the many possible states to one state. It has been related to measurements (the Copenhagen interpretation) or the involvement of consciousness (which is in itself not well understood). Attributing the reduction to consciousness "seems to assign a privileged role to those corners of the Universe where consciousness resides-and they may be very rare indeed" (Penrose 1987a, 33).

Penrose thinks that quantum mechanics, with its linear superposition, has to be replaced by a nonlinear theory, which perhaps would not have these problems. This would be similar to the transition from Newton's theory of gravitation, which has the total gravitational force as a linear superposition of the forces contributed by the sources, to Einstein's general theory of relativity, which is "an astonishing non-linear theory of even greater mathematical elegance" (Penrose 1987a, 34). Penrose has not formulated that theory (yet), but he offers a suggestion. The "linear superposition of states will cease to be maintained by nature as soon as the states become significantly differently coupled into the gravitational field"' (Penrose 1986b, 50). "The idea is that, in a sense, Nature abhors complex linear combinations of differing spacetime geometries!' (Penrose 1987a, 44). This seems to have testable consequences, like a prediction of minimal bubble sizes in a bubble chamber-a measuring device to detect tracks of elementary particles (Penrose 1986a, 144). The theory will be "non-local in a way that fundamentally affects even the very fabric of spacetime. I do not expect, except perhaps at some temporary provisional level, that we shall be able to get away with a theory which describes objective reality taking place within some ambient spacetime"' (Penrose 1987a, 45).

Penrose (1987b, 1989) even suggested that this approach might result in understanding the nature of consciousness. Life thrives on minute effects in chemistry. Similarly, Penrose speculates, consciousness might be a phenomenon that thrives on minute effects in physics which happen at the transition from the quantum realm to the realm describable by classical physics.

\section{IMPLICATIONS FOR SCIENCE AND THEOLOGY}

These three very different approaches make it seem that cosmology is open to metaphysical influences, especially from this representa- 
tive diversity at the frontier of cosmology. Thus, as will be discussed below, this could be one of the few places where religious positions might actually and rightly influence scientific research, by influencing its metaphysical choices. The limits of the Big Bang theory are very relevant to its use in cosmological arguments for the existence of God. The diversity and the lack of scientific consensus also have implications for the way theology should deal with science, at least with cosmology, as we will see.

Metaphysics and the Variety of Cosmologies. According to a philosopher of science, Imre Lakatos, one can describe science as consisting of various research programs. ${ }^{8}$ Such programs are more or less coherent series of theories, characterized by a hard core-the hypotheses that are kept fixed. Other hypotheses can be added or changed, according to theoretical or empirical needs, to form a protective belt around the core. It is in this outer area that one finds the major development within a program. The development is not haphazard but is guided by a long-term research policy: the positive heuristic. Theoretical science has a more or less autonomous development, guided more by the awareness of the unsatisfactory character of the theory at each moment than by specific, experimental results.

Although we did not discuss the development of cosmologies, they exhibit these same characteristics as a series of articles which modify their ideas within a basic continuity (Drees 1990b). Our cosmologists agree for their hard core on the validity of the Big Bang theory after "the first fraction of a second" and on what can be called the standard problems of this model. They also agree that the Copenhagen interpretation of quantum mechanics is unsatisfactory, since the notion of an observer makes no sense for the Universe as a whole. However, these cosmologies are nonetheless rather different, especially with respect to time and quantum reality, and therefore have different problems to solve. Penrose needs to explain how reality becomes classical through a natural reduction of the state vector, and Hawking needs to explain why observers see a definite universe instead of the superposition of many states that is his view of reality. Penrose must also explain why his time asymmetry is mostly hidden-in other words, why time-symmetric physics (Newtonian, relativistic, and quantum physics) works so well. For Hawking, asymmetries need to be explained on the basis of a symmetric theory and symmetric boundary conditions. Implicitly, these cosmologists disagree about the data. For Penrose, the time-asymmetric phenomena are real. For Hawking, time asymmetry is, in principle, an 
illusion. His aim is not to incorporate the asymmetry, but to explain time-asymmetric phenomena away.

According to Lakatos, "one may formulate the 'positive heuristic' of a research program as a 'metaphysical' principle" (Lakatos 1970 $[1978,51])$. Lakatos uses metaphysical "as a technical term of naive falsificationism: a contingent proposition is 'metaphysical' if it has no 'potential falsifiers' "' (Lakatos 1970 [1978, 47, n. 2]).

For the cosmological programs discussed in this article, the ideas are not "metaphysical" only in the sense that they are beyond dispute, at least each within its program, they are also metaphysical in the classical sense, as they are about such issues as the relation between potential and actual existence, the nature of space and time, the (dis)similarity of future and past, and the contingency or necessity of the universe. These metaphysical elements are most clearly visible in the positive heuristic as ideas guiding the direction of the research, and hence influencing the next step in the development of the program.

Science is in general leading our understanding of the world. However, metaphysical influences in constructing even the most abstract theories about our Universe provide an opportunity for religious influence on scientific research. If one has a strong theological interest in history, one would prefer a metaphysics which incorporates time asymmetry. Hence, one might prefer to work in a program which fits that interest, as Penrose's program does. If one's religion is more about God as the ultimate, atemporal, transcendent Ground, one might prefer something like Hawking's approach. Metaphysical diversity on such issues as the nature of time and reality means that one can achieve consonance in some cases by opting for that scientific program which best fits one's theological ideas.

I am not claiming that all scientists have such religious convictions. Nor do I mean to imply that theologians should give the scientists advice; the work of the scientists is far too complex. But I suggest that the religious convictions of a scientist might be reflected in the research that he or she pursues. A professional theologian or philosopher might, perhaps, be of some help by making as explicit as possible the substance and metaphysical background of such convictions. Also, the fact that there is a plurality of programs in cosmological research beyond the Big Bang theory might have implications for the way one can relate the results of science to theology. 
Cosmological Arguments for the Existence of God. The temporal cosmological argument for the existence of God on the basis of a beginning of the Universe (e.g., Craig 1979) turns out not to be univocally supported by science as research progresses beyond the Big Bang theory. Some theories have a beginning, others are without an absolute beginning (e.g., Linde's), and still other theories present a view in which the "beginning" loses its special status as a feature of reality (e.g., Hawking's). It is not amazing that theologians and philosophers are not informed about current cosmological research, but it is a serious failure if one neglects the limits of the theories one uses.

A nontemporal version of the cosmological argument, which claims that the whole series of states, whether finite or infinite, needs an explanation, has been defended by, among others, Richard Swinburne (1979). The present research does not affect his argument that ultimately we have to choose between two possibilities: "the universe as a stopping-point and God as a stopping-point"' (Swinburne 1979, 127). However, his argument for preferring God as the stoppingpoint rests on the claim that "God" is a much simpler assumption than the Universe, with all its complexities, and this assumption might be doubted from the perspective of contemporary cosmology. Theories about the Universe gain continuously in elegance and simplicity-if not in the calculations, at least in their structure and assumptions - and hence those theories might be a simpler stopping-point than "God."

Theology and the Lack of Consensus in Science. Some in "science and religion" focus on methodological issues, but science is also theologically relevant for its content. For instance, Hawking has a quite different view of the nature of time than Penrose. This implies different views of history and processes, which bear upon many theological issues.

Many authors, in contributing to "science and religion," restrict themselves to the areas where science shows consensus. However, this is much less secure than it seems. For instance, the Big Bang theory suggests a certain view of the "beginning" of the Universe, but a more detailed study of scientific research shows that the interesting issues are not in the Big Bang theory but just beyond its limits, in current research. And, even worse, though the different programs accept the Big Bang theory, they disagree on many issues of its interpretation because they envisage a different, further development of the scientific understanding of the universe. This raises the question of the relevance of the established scientific theories for theology.

If one leaves the domain of consensus, one has to face the plurality 
of approaches at the leading edge of research. This is not only the case for cosmology, but holds as well for other areas of fundamental physics-for instance, the interpretation of quantum mechanics or thermodynamics (see, e.g., Russell 1988, 370).

Should one in "science and theology" restrict oneself to the most promising ideas (but how does one know?), pick the idea that best fits one's personal view (but that is rather selective), or wait till there is consensus (which might take a long time)? In my opinion, intelligible models may be developed in the context of as many approaches as feasible, with the intention of learning from each what one can and cannot say meaningfully about theological concepts within that scientific program. However, once one is after plausibility, or even truth, persistent plurality becomes more problematic. We should be willing to face that scientific program that is the hardest to encompassa kind of reverse eclecticism: take science "where it 'hurts' most" (Eaves 1989, 203). We may have to accept the provisional nature and uncertain status of all theological models, even if they have been built upon the available scientific consensus.

\section{EDGES, CREATION, AND NOTHING}

In this section we will focus on the notion of creation out of nothing in the context of such cosmologies as that of Hawking. The absence of a beginning of time in some theories has been taken by some to imply the absence of a Creator. It will be argued that this rests on a view of God and God's role in creation which is not the view of most developed theologies. Our second issue is the claim that physical cosmology is now able to describe creation out of nothing. It will be argued that this physical concept of nothing is not identical to the philosophical concept.

Edges and Deism. For Carl Sagan the issue is simple: If Hawking is right, that there is no absolute beginning of reality, there is no need for a Creator. This is quite similar to the argument of others, that a beginning in the Big Bang sense supports belief in a Creator. However, a good number of theologians and others have argued that the two-a physical beginning and belief in God-are mutually independent.

One could have an image of God as the great watchmaker who constructed the world and wound the spring-before letting it run by itself according to the original design. In relation to such an image of God the beginning is very important, for it is there that the watchmaker did his (or her) job. This kind of theological imagery has been, 
and perhaps still is, widespread, as well as that of God as "an Old Man up there." Belief in the watchmaker-God has been labeled deism, in contrast to theism, which holds that God is actively involved in the processes of the world at later moments. Contemporary theologians have in many different ways argued that it is of major importance to see God as related to the present. A purely Deistic concept of God is not a serious option within contemporary theology, because such a God would not be relevant to us and the ways we shape our lives.

Removal of a beginning would imply that the watchmaker God is not a defensible image. That seems to be the essence of Sagan's remark. However, that is not a deathblow to theism, as it is not the kind of God theism defends. For some theologies it is even the other way round. For instance, process theology, a theological strand which developed on the basis of the philosophies of Whitehead and Hartshorne, argues that there is no absolute beginning but only an eternal process in which the world and God exert influence on each other (e.g., see Cobb and Griffin 1976). This particular theology does not fit the Hawking theory either, because their views of the nature of time are very different. But the example of such a theology, which is intellectually well developed, shows that theology is not necessarily bound to an absolute beginning, an edge to time.

Whether it is possible to develop a theological view which fits the whole picture, not only the "edgelessness" but also the nature of time, the determinism, and the completeness suggested in the Hawking cosmology, remains to be seen. But Sagan's argument, "No edge, hence no God," is not decisive. Aside from the possibilities which might still be present in the context of the Hawking cosmology, one also needs to keep in mind the status of this cosmology. As argued above, there is a genuine pluralism in contemporary cosmology, and this pluralism is most significant where the cosmologies touch on the most fundamental metaphysical questions. Hence, one could opt for a cosmology which allows for such an edge, a beginning of time, as Penrose's cosmology does.

Out of Nothing. Hartle and Hawking interpreted their proposal for the wave function of the universe as conceding the probability "for the universe to appear from Nothing" (Hartle and Hawking 1983 , 2961). In this section I will argue that the Hartle-Hawking theory does not describe such an appearance-out-of-nothing, if that notion is taken in its absolute sense. Neither do other theories, such as those of Vilenkin. In the subsequent section I will argue that the Hartle-Hawking theory can be interpreted in the sense of creatio 
ex nihilo, but that this theological notion should then be understood as a view of the universe as sustained by God at every moment, rather than as a cosmogonic expression.

There is one sense in which Hawking's theory can most clearly be understood as creation from "nothing." As was explained above, ordinary calculations often assume a state at one moment and laws to calculate the state at another moment. One might say that the second state arises out of the first state. There is in the HartleHawking approach at the timeless level no reference to a state other than the "resulting" state. Because it is compact, it is the only boundary in the calculation. The theory gives a precise meaning to the notion of nothing as the absence of other boundaries in the calculation. However, this should not be misunderstood as appearance-out-of-nothing. Appearance is a temporal notion, while "from nothing"' applies to a time-independent actuality.

The "nothing" is not an absolute nothing. One "must still grant the existence of quite a body of pre-existing laws of Nature in order to get away with this trick" (Barrow 1988, 231; similarly Heller 1987, 421) - for example, quantum laws and fields as well as mathematical logic. The "nothing" which has a precise meaning in the context of this proposal is not an absolute "nothing" in a more philosophical sense.

There are serious problems when one tries to combine the language of probabilities with the notion of nothing:

1. The probability of "heads" when tossing a coin is fiftyfifty-but there is a 50 percent chance of actually getting "heads" if and only if, someone tosses a coin; that is, if, and only if, one of the possible outcomes is actualized. A mathematical idea of getting a universe from nothing will not produce a physical universe, but only the idea of a physical universe-assuming that there is a difference between the universe and a mathematical idea about the universe. There has to be some input of "physical reality." Perhaps that is an aspect of the nothingness, but that aspect makes it into a physical entity and not into nothing at all.

2. Physical probabilities, as exemplified by radioactive decay, start with something, with an initial situation (a particle in space and time) becoming another situation (other particles in space and time). The probability is that the transition from situation 1 to situation 2 happens during a certain interval of time, or that the particle is found in a certain volume of space, or something of that nature. Even if one reduces the entities in the first situation as much as possible (no energy, no matter fields, etc.), talking about probabilities makes 
sense only if there is some measurable structure (such as time) in the first situation.

Interpreting the Hartle-Hawking wave function as giving probabilities for appearance-out-of-nothing is too strong. More defensible and modest, rather is the claim that it "determines the relative probability of universes corresponding to different classical solutions"' (Hawking 1984a, 377).

Creation as Cosmogony or Dependency. Traditional theological ideas about creatio ex nihilo have two poles. On the one hand they refer to cosmogony, the coming into being of our universe. On the other hand they denote eternal sustaining by God, ultimate dependence at each moment. Chris Isham states that the latter concept "is somewhat decoupled from modern scientific thought" (Isham 1988, 376), a view that is probably widely shared among modern theologians and scientists. However, a recent exception is Pannenberg, who argued that the notion of "inertia" (or mass) in physics is at odds with the theological idea that the world is continuously sustained by God (Pannenberg 1981, 1988).

In my view, the Hawking cosmology lends itself more to an interpretation in terms of sustaining rather than making. The basic entities are three-dimensional spaces with their material content (fields). Therefore, these are to be seen in this context as the basic entities of creation, the "what" that is created, and their relative probabilities can be calculated on the timeless level. It is not that one results from the other or comes after the other. From the timeless perspective, they are all coeternal, or they are all created "timelessly." Hence they all are equally related to the Ground of Being. Another way to argue for the same conclusion is to say that this scheme does not have an initial event with a special status. There is no way to pick one slice as the first of the sequence. Hence, all moments have a similar relation to the Creator. Either they are all "just brute facts" or they are all equally created. This view of God "sustaining" the world in all its "times" was transformed by Isham into another image: "One can almost imagine the universe .. . being held in the cup of God's hand"' (Isham 1988, 405).

To summarize, this theory allows for a precise interpretation of ex nihilo, and it fits better with the idea that every space (with content) is equally created by God than with the idea that God created "the beginning." Questions about the relation between the two components of creatio ex nihilo follow. Understanding creation as a cosmogonic process seems to single out a certain event as having a special relation to God, whereas sustaining tends to stress the 
similarity of all states in their relation to God. Theologians who want to defend both components of creatio ex nihilo need to clarify the similarity and dissimilarity between the first and the later states in their relation to God. In the theory discussed here, no moment has a special status, and therefore the cosmogonic interpretation loses its force.

Theology in the Context of Hawking's Cosmology. The cosmologies described in this article provide different contexts for theological thought. A theology that fits one cosmology need not accord with the other cosmological programs. Hawking's cosmology, as one of the well-developed contemporary programs, does not fit theologies that put a strong emphasis on processes in time. Amazingly, his quantum cosmology seems much closer to two seventeenthcentury views and might be made consonant with traditional Reformed theology, which saw everything as predetermined by God. Also, it might be combined with a Spinozistic view of God and the world, where the world is one of God's eternal modes of being. As does the traditional reformed view, Hawking's approach accepts strict determinism. In a sense, however, the Spinozistic view fits even better, as the Universe acquires in Hawking's cosmology some of God's characteristics, being timeless, eternal, and "necessary."

In case one tries to combine Hawking's cosmology with a Christian view of the world, there is one major difficulty. As presented here, his theory is about three-dimensional spaces with material content. They may be ordered in time-sequences, but that is secondary. Therefore, Hawking's model lacks continuity between subsequent events, like the continuity we find in reading the first and then the second word of a sentence. ${ }^{9}$ One might evoke God as the One who gives continuity, but that makes God "close the gaps," to turn a phrase. In the major Christian traditions there is a sense of continuity, as God's great deeds in the past (e.g., in Exodus, the covenant on Sinai, the Incarnation, the Resurrection) are supposed to have relevance for today and for the future. In a Christian perspective, the past has to be taken into the present in some way.

A final word of caution: this theory is not the conclusion of science today. It is still in development, and it is only one program among others, although one of the most elegant and coherent. The special feature of the Hartle-Hawking proposal is the absence of boundaries, its specific form of ex nihilo. 


\section{NOTES}

1. The discussion of recent developments in science has to be very incomplete. At a popular level, more can be found in Pagels (1985), Trefil (1983), Davies (1984), and other books as well as in the article by Odenwald (1989). Convenient access to current research can be gained from conference proceedings.

2. Energy is conserved during "inflation." Binding energy, provided by the attractive force of gravity, is negative since it costs energy to free constituents. Gravitational energy becomes even more negative due to the inflationary expansion. This provides energy that is converted into radiation and particles when the inflationary expansion ends.

3. Linde accepts the existence of singularities but argues that they may be more or less local phenomena and hence do not imply a beginning for the whole Universe (see Linde 1987a, 619).

4. Imaginary must not be misunderstood as opposed to factual; it is a technical term for numbers which, by definition, give a negative result when squared. "Distances" in space-time $\left(d^{2}=x^{2}+y^{2}+z^{2}-c^{2} \cdot t^{2}\right)$ combine the spatial coordinates $x, y$, and $z$ with the time coordinate $t$, but they aren't on a par. The minus sign has enormous consequences-for instance, solutions in general relativity may be spatially finite without edges (like the surface of a sphere), but with edges in time, have a beginning and end. Due to the properties of imaginary numbers, the minus sign disappears if one takes an imaginary coordinate $t$. This allows for solutions without edges, whether in space or "time," which now is that imaginary time variable.

Hawking seems to hold that we perceive the universe in real time, and thus with a beginning and end, but that the universe exists in imaginary time and hence without Singularities, without a beginning and end. "But . . a scientific theory is just a mathematical model we make to describe our observations: it exists only in our minds. So it is meaningless to ask: Which is real, 'real', or 'imaginary' time? It is simply a matter of which is the more useful description" (Hawking 1988, 139). The appeal to usefulness seems more instrumentalistic than in Hawking's other writings-for instance, about the beginning (or its nonexistence) and God.

5. It can be found in the eleventh book of Augustine's Confessions. A similar idea had also been expressed a few centuries earlier by Philo of Alexandria, who even thought that time might start after the creation of the material world, when movement would begin. See sec. 26 of On the Account of the World's Creation by Moses by Philo of Alexandria.

6. Penrose's cosmological ideas have their background in the twistor program (Penrose 1972; Penrose and Rindler 1984 and 1986). We will not go into that part of his work; it is an original but not easily accessible approach. In short, Penrose holds that twistors represent a level of reality deeper than that of particles and space-time points.

7. If there is a subsystem, the whole wave function is as the product of wave functions for the subsystem and for "everything else." A measurement of the subsystem changes the first term in this product, but "everything else" remains the same (Tipler 1986, 247). This is in line with Hugh Everett's original proposal (Everett 1957). The understanding of the Many Worlds Interpretation as if everything else also splits is more or less due to Bryce DeWitt (DeWitt and Graham 1973).

8. Lakatos's methodology of scientific research programs (1970) has been applied both to theology and to religion and science-for example, by Nancey Murphy (1987; 1990) and Hefner (1989).

9. The existence of two levels of description, one timelessly viewing the whole of time at once and the other from within time, is quite common in physics. For example, systems can be described as evolutionary, but also by trajectories in phase space. These trajectories represent complete possible histories of the system. In Big Bang cosmology the four-dimensional space-time can be described as a three-dimensional spatial universe expanding in time. The peculiar feature of Hawking's cosmology is that the timeless description is not about the whole history of time, but about individual slices of "now." In that sense, this timeless description undermines the continuity between subsequent 
slices more than do other timeless theories in physics. This might change because of future developments or reinterpretations of the theory.

\section{REFERENCES}

Barrow, J. D. 1988. The World within the World. Oxford: Clarendon Press.

Bell, J.S. 1981. "Quantum Mechanics for Cosmologists." In Quantum Gravity 2, ed. C.J. Isham, R. Penrose, and D. W. Sciama. Oxford: Clarendon Press.

Boslaugh, J. 1985. Stephen Hawking's Universe. New York: Quill/William Morrow.

Cobb, J. B., and D. R. Griffin. 1976. Process Theology: An Introductory Exposition. Philadelphia: Westminster Press.

Craig, W. L. 1979. The Kalam Cosmological Argument. London: Macmillan.

Davies, P.C.W. (Paul). 1984. Superforce. New York: Simon and Schuster.

DeWitt, B.S., and N. Graham, eds. 1973. The Many-Worlds Interpretation of Quantum Mechanics. Princeton, N.J.: Princeton Univ. Press.

Drees, W. B. 1990a. Beyond the Big Bang: Quantum Cosmologies and God. La Salle, Ill.: Open Court.

. 1990b. "Philosophical Elements in Penrose's and Hawking's Research in Contemporary Cosmology." Philosophy in Science 4:13-46.

Eaves, L. 1989. "Spirit, Method, and Content in Science and Religion: The Theological Perspective of a Geneticist." Zygon: Journal of Religion and Science 24 (June): 185-215.

Everett, H. 1957. "'Relative State' Formulation of Quantum Mechanics." Reviews of Modern Physics 29:454-65.

Gibbons, G.W., S.W. Hawking, and J. M. Stewart. 1987. “A Natural Measure on the Set of All Possible Universes." Nuclear Physics B 281:736-51.

Hartle, J. B., and S. W. Hawking. 1983. "Wavefunction of the Universe." Physical Review D 28:2960-75.

Hawking, S. W. 1980. Is the End in Sight for Theoretical Physics? Cambridge: Cambridge Univ. Press. Reprinted in J. Boslaugh. 1985. Stephen Hawking's Universe. New York: Quill/William Morrow.

- _ 1984a. "Quantum Cosmology." In Relativity, Groups, and Topology II, ed. B.S. DeWitt and R. Stora. Amsterdam: North Holland.

- 1984b. "The Edge of Spacetime.", New Scientist 103 (16 Aug): 10-14.

. 1984c. "The Edge of Spacetime." American Scientist 72:355-59.

- 1985. "Arrow of Time in Cosmology." Physical Review D 32:2489-95.

. 1987. "Quantum Cosmology." In Three Hundred Years of Gravitation, ed. S.W. Hawking and W. Israel. Cambridge: Cambridge Univ. Press.

1988. A Brief History of Time. New York: Bantam Books.

Hawking, S. W., and D. N. Page. 1986. "Operator Ordering and the Flatness of the Universe." Nuclear Physics B 264: 185-96.

Hawking, S. W., and R. Penrose. 1970. "The Singularities of Gravitational Collapse and Cosmology." Proceedings of the Royal Society of London A 314:529-48.

Healey, R. A. 1984. “'How Many Worlds?” Nous 18:591-616.

Hefner, P. 1989. "The Role of Science in Pannenberg's Theological Thinking." Zygon: Journal of Religion and Science 24 (June): 135-51.

Heller, M. 1987. "Big Bang on Ultimate Questions." In Origin and Early History of the Universe: Proceedings of the 26th Liegge International Astrophysics Colloquium, July 1-4 1986. Cointe-Ougree (Belgium).

Isham, C.J. 1988. "Creation of the Universe as a Quantum Process." In Physics, Philosophy, and Theology: A Common Quest for Understanding, ed. R.J. Russell, W. R. Stoeger, and G.V. Coyne. Vatican City: Vatican Observatory; distributed in U.S. by Univ. of Notre Dame Press, Notre Dame, Ind.

Lakatos, I. 1970. "Falsification and the Methodology of Scientific Research Programs." In Criticism and the Growth of Knowledge, ed. I. Lakatos and A. Musgrave. Cambridge: Cambridge Univ. Press. Reprinted in I. Lakatos. 1978. Philosophical Papers, Vol. 1, ed. J. Worrall and G. Currie. Cambridge: Cambridge Univ. Press. 
Linde, A. D. 1985. "'Particle Physics and Cosmology." Progress of Theoretical Physics, Supplement 85:279-91.

- 1987a. "Inflation and Quantum Cosmology." In Three Hundred Years of Gravitation, ed: S.W. Hawking and W. Israel. Cambridge: Cambridge Univ. Press.

C. 1987b. "Particle Physics and Inflationary Cosmology." Physics Today $40(9): 61-68$.

. 1989. "Life after Inflation and the Cosmological Problem." Physics Letters B 227:352-58.

Murphy, N. 1987. “Acceptability Criteria for Work in Theology and Science." Zygon: Journal of Religion and Science 22: 279-97.

- 1990. Theology in the Age of Scientific Reasoning. Ithaca, N.Y.: Cornell Univ. Press.

Odenwald, S. F. 1990. " A Modern Look at the Origin of the Universe." Zygon: Journal of Religion and Science 25 (March): 25-45.

Pagels, H.R. 1985. Perfect Symmetry: The Search for the Beginning of Time. New York: Simon and Schuster.

Pannenberg, W. 1981. "Theological Questions to Scientists." Zygon: Journal of Religion and Science 16: 65-77. Also in The Sciences and Theology in the Twentieth Century, ed. A. R. Peacocke. South Bend, Ind.: Univ. of Notre Dame Press.

-1988. "The Doctrine of Creation and Modern Science." Zygon: Journal of Religion and Science 23:3-21.

Penrose, R. 1972. "'On the Nature of Quantum Geometry." In Magic without Magic: John Archibald Wheeler, ed. J. R. Klauder. San Francisco: W. H. Freeman.

-1979. "Singularities and Time-asymmetry." In General Relativity: An Einstein Centenary Survey, ed. S.W. Hawking and W. Israel. Cambridge: Cambridge Univ. Press.

-. 1981. "Time Asymmetry and Quantum Gravity." In Quantum Gravity 2, ed. C.J. Isham, R. Penrose, and D. W. Sciama. Oxford: Clarendon Press.

- 1986a. "Gravity and State Vector Reduction." In Quantum Concepts in Space and Time, ed. R. Penrose and C.J. Isham. Oxford: Clarendon Press. . 1986b. "Herman Weyl, Space-time and Conformal Geometry." In Herman Weyl, 1885-1985, ed. K. Chandrasekharan. Berlin: Springer Verlag.

- 1987a. "Newton, Quantum Theory, and Reality." In Three Hundred Years of Gravitation, ed. S. W. Hawking and W. Israel. Cambridge: Cambridge Univ. Press.

- . 1987b. "Quantum Physics and Conscious Thought." In Quantum Implications, ed. B.J. Hiley and F. D. Peat. London: Routledge and Kegan Paul.

. 1989. The Emperor's New Mind. Oxford: Oxford Univ. Press.

Penrose, R., and W. Rindler. 1984, 1986. Spinors and Space-time. 2 vols. Cambridge: Cambridge Univ. Press.

Russell, R.J. 1988. "Quantum Physics in Philosophical and Theological Perspective." In Physics, Philosophy, and Theology: A Common Quest for Understanding, ed. R.J. Russell, W. R. Stoeger, and G.V. Coyne, Vatican City: Vatican Observatory; distributed in U.S. by Univ. of Notre Dame Press, Notre Dame, Ind.

Sagan, C. 1988. Introduction to A Brief History of Time, by S. W. Hawking. New York: Bantam Books.

Shimony, A. 1986. "Events and Processes in the Quantum World." In Quantum Concepts in Space and Time, ed. R. Penrose and C.J. Isham. Oxford: Clarendon Press.

Stein, H. 1984. "The Everett Interpretation of Quantum Mechanics: Many Worlds or None?" Nous 18:635-52.

Swinburne, R. 1979. The Existence of God. Oxford: Oxford Univ. Press.

Tipler, F.J. 1986. "Interpreting the Wavefunction of the Universe." Physics Reports 137:231-75.

- 1989. "The Omega Point as Eschaton: Answers to Pannenberg's Questions for Scientists." Zygon: Journal of Religion and Science 24 (2): 217-53. 
Trefil, J.S. 1983. The Moment of Creation: Big Bang Physics from before the First Millisecond to the Present Universe. New York: Scribner's.

Turner, M. 1985. "The Inflationary Paradigm." In Fundamental Interactions and Cosmology, ed. J. Audouze and J. Tran Thanh Van. Gif sur Yvette: Editions Frontières (France).

Vilenkin, A. 1986. "'Boundary Conditions in Quantum Cosmology." Nuclear Physics B 252:141-51.

Weinberg, S. 1977. The First Three Minutes. New York: Basic Books.

\section{Pre-Publication Discount Offer! SAVE $15 \%$}

\section{THE FIRST BOOK-LENGTH STUDY OF ONE OF THE 20TH GENTURY'S SEMINAL THINKERS}

Yoking Science and Religion:

The Life and Thought of Ralph Wendell Burhoe by David R. Breed Foreword by Roger W. Sperry (Nobel Laureate, brain sciences)

Publication Date: February 1, 1992

Pre-publication price - $\$ \mathbf{1 0 . 9 5}$

After January $15,1992-\$ 12.95$

Mail orders (check) or telephone orders (VISA/Master Card) to:

LSTC BookCenter, 1100 E, 55th St., Chicago, IL 60615-5199, USA: Telephone (312) 753-0753.

This offer good only for check or credit card orders!! 